\section{Pulmonary rehabilitation; what's in a name?}

\author{
Sally J Singh, Michael C Steiner
}

The therapeutic efficacy of pulmonary rehabilitation is now well established and supported by a substantial body of clinical trial evidence. ${ }^{12}$ The place of pulmonary rehabilitation in the management of chronic obstructive pulmonary disease (COPD) and other chronic respiratory diseases has been enshrined in national and international guidelines including those recently produced by the British Thoracic Society. ${ }^{3}$ In recent years, attention has shifted from questions regarding the effectiveness of the intervention for those who successfully attend and complete a programme, to meeting challenges for the delivery of pulmonary rehabilitation to the wider population with disabling lung disease. A key driver of this focus is the perception in many quarters that uptake and adherence to rehabilitation is poor, and that a significant minority of patients do not fully respond, or quickly decline, once the programme is completed. As a result, there is considerable interest in developing and testing alternative delivery models of pulmonary rehabilitation and other behavioural interventions that aim to improve general health, knowledge of the disease and promote self-care.

Casey et $a l^{4}$ presents data from the PRINCE study which reports on the delivery of a structured education pulmonary rehabilitation programme (SEPRP) in a primary care setting. This is not the first study of pulmonary rehabilitation in the community, ${ }^{6}$ but the PRINCE study has taken an alternative approach. The authors describe a tightly controlled cluster ramdomised controlled trial (RCT) of a rehabilitation intervention versus best usual care in a group of participants with moderate to severe COPD. The authors should be congratulated on conducting such a rigorous randomised controlled trial in a large study population. Participants underwent an 8-week programme, comprising weekly sessions of exercise and education,

Centre for Exercise and Rehabilitation Science, Department of Respiratory Medicine, University Hospitals of Leicester NHS Trust, UK

Correspondence to Dr Michael Steiner, Centre for Exercise and Rehabilitation Science, Department of Respiratory Medicine, University Hospitals of Leicester NHS Trust, Groby Road, Leicester LE3 9QP, UK; michael.steiner@uhl-tr.nhs.uk followed by a telephone call at 4 weeks postdischarge, and a $1 \mathrm{~h}$ group meeting at 12 weeks. The overall results, at 12 weeks were mixed; the primary outcome measure, the chronic respiratory disease questionnaire did achieve between group statistical significance, but the confidence intervals overlapped with the minimal clinically important difference for the measure leaving doubts about the importance of the population effect. This lack of clarity in this outcome is in part due to an improvement in health status in the control group, a frequently observed phenomenon in clinical trials. However, the intervention also failed to influence secondary outcomes including exercise tolerance and, overall, these results would have to been seen as rather disappointing when compared with conventional pulmonary rehabilitation and, indeed, to other recent community-based trials. ${ }^{5} 6$ The authors suggest their population might have been 'too good', but although their mean performance on the incremental shuttle walking test (ISWT) was slightly higher than has previously been described, ${ }^{7} 8$ values were well below the predicted normal range. ${ }^{9}$ Similarly, while the responsiveness of exercise testing may vary between populations and between the exercise outcomes frequently reported, there is a substantial body of evidence to suggest that the ISWT is responsive to rehabilitation across a range of disease severities ${ }^{10-12}$ including less disabled patients. ${ }^{11} 13$ We think it more likely that there was a failure to intervene rather than a failure of the chosen outcome measures to detect an effect.

The novelty of the paper is the approach taken with the educational component. Practice nurses, who potentially have limited knowledge about COPD, were enrolled on a 3-day course on adult education to equip them to deliver the educational package. However, there is now recognition that a collaborative approach to the educational component of rehabilitation, promoting and negotiating behaviour change is needed rather than a more traditional didactic approach. This may require more experience of the rehabilitation process on the part of the practitioner and an appreciation of motivational techniques. Equally, it has been a long held premise of rehabilitation that the programme should be delivered by a multidisciplinary team, and it is usually the educational component that reflects this. The programme would probably have benefitted from wider team involvement, most obviously an occupational therapist and dietician. The education programme appears to have been precisely defined, whereas the exercise programme was more loosely prescribed and did not meet the specified levels described in national or international guidelines where the current recommendation is for at least two supervised sessions a week, with accompanying unsupervised home training sessions. ${ }^{3} 14$

When considering the delivery of pulmonary rehabilitation, there are two perspectives: that of the individual participating and that of the provider organisation. Both would want to facilitate better access to the service but may have a different outlook on the setting, content, supervision and cost of the programme. What, therefore, are the lessons that can be drawn from the PRINCE trial? The aim of pulmonary rehabilitation is to take a disabled patient with individual needs and priorities and provide an individually tailored intervention that improves symptoms and maximises physical and social functioning. The components and setting of rehabilitation may vary, but supervised exercise training conducted at least twice a week has consistently been shown to be the key to its effectiveness. It is possible that the supervision of the intervention that is required to bring about these objectives will vary considerably between patients and, therefore, home-based pulmonary rehabilitation or self-managed exercise programmes may offer an effective alternative for some people. Indeed such innovations may improve access to treatment for some (eg, patients who are still at work) and create capacity for more disabled patients to access formal programmes. Despite reservations about the 'dose' of pulmonary rehabilitation provided in the PRINCE trial some individuals might prefer the style and setting of a 'non-traditional' intervention, and may accrue important benefits which they might be denied if the only format offered is felt to be unmanageable or impractical by the patient. Offering a diversity of patient treatment choices is self-evidently desirable but this needs to be an informed choice of effective treatment options. One concern with less intensely supervised interventions is that patients may collude in the avoidance of a treatment whose benefits are at least 


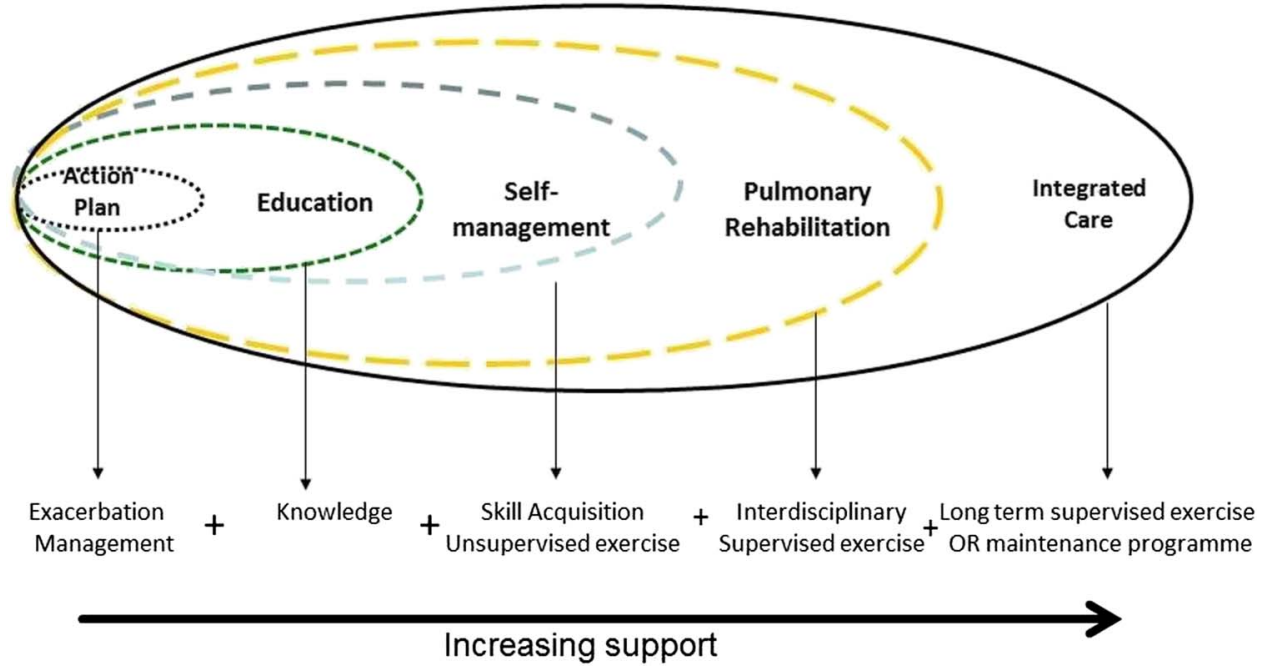

Figure 1 The spectrum of Rehabilitation and Self-Management interventions in Chronic Disease Management Programmes for COPD (adapted from Wagg $^{16}$ ).

in part dependent on their own individual effort and motivation. Such informed decision making needs to be considered alongside concepts of supported selfmanagement which are central to modern care of long-term conditions. There is evidence that self-management interventions will be effective if they are properly supported and embedded in wider chronic disease management programmes, for example, the Chronic Care Model, ${ }^{15}$ and pulmonary rehabilitation and supported self-management are key components of such programmes (figure $1^{16}$ ). For example, the PRINCE intervention, and indeed any pulmonary rehabilitation intervention, would provide aspects of education, self-management training and supervised exercise.

So what should we call pulmonary rehabilitation and what is selfmanagement? There appears to be a great deal of overlap in the use of this terminology, but one of the defining features of a pulmonary rehabilitation programme is an accurately prescribed exercise programme, with clear markers of progression. To ensure a high-quality service that adheres to national guidance with adequately trained staff, the process of rehabilitation will need to be quality controlled; indeed, some countries have a system of accreditation in place. This process would not seek to limit the delivery of alternative forms of rehabilitation, but there is a threshold which needs to be met based on clear guidance about how to maximise the chance of a beneficial response to the exercise training regime before a programme should be termed pulmonary rehabilitation. These criteria might need to be tempered for different disease or clinical settings (eg, posthospitalisation for acute exacerbation) but quality assurance of individual programmes can be underpinned by rigorous audit of clinical outcomes. Ideally, the facility to stratify and personalise the intensity and setting of support for individual patients would be built into the care provided. Personalised therapy is clearly desirable but requires the tools to support stratification and individual decision making. In the case of pulmonary rehabilitation and self-management programmes, this would require an assessment of a patient's personal resources, education and motivation as well as physical characteristics and disease severity. An understanding of these individual issues would allow the rehabilitation practitioner to titrate the intensity and character of the support provided according to individual needs.

Clinicians and rehabilitation practitioners frequently encounter patients who decline the offer of pulmonary rehabilitation or fail to stay the course. However, in truth, adherence and response rates are comparable to many other health interventions including drug therapies, and despite these frustrations, pulmonary rehabilitation is of clear clinical and economic value. ${ }^{17} 18$ The challenge for the pulmonary rehabilitation community is diversifying patient choice so that access and uptake of therapy is maximised without sacrificing quality and efficacy.

Contributors Both authors contributed equally to the writing of the article.

\section{Competing interests None.}

Provenance and peer review Commissioned; internally peer reviewed.

To cite Singh SJ, Steiner MC. Thorax 2013;68: 899-901.
Published Online First 10 July 2013

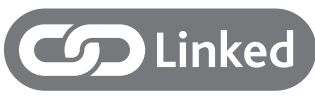

http://dx.doi.org/10.1136/thoraxjnl-2012-203103

Thorax 2013;68:899-901.

doi:10.1136/thoraxjnl-2013-203716

\section{REFERENCES}

1 Lacasse Y, Goldstein R, Lasserson TJ, et al. Pulmonary rehabilitation for chronic obstructive pulmonary disease. Cochrane Database Syst Rev 2006;(4):CD003793.

2 Griffiths TL, Burr ML, Campbell IA, et al. Results at 1 year of outpatient multidisciplinary pulmonary rehabilitation: a randomised controlled trial. Lancet 2000;355:362-8.

3 The British Thoracic Society Guideline on Pulmonary Rehabilitation in Adults. Thorax 2013;68 (Supp 2):1-30.

4 Casey D, Murphy K, Devane D, et al. The effectiveness of a structured education pulmonary rehabilitation programme for improving the health status of people with moderate and severe chronic obstructive pulmonary disease in primary care: The PRINCE cluster randomised trial. Thorax 2013:68:922-8.

5 Waterhouse JC, Walters SJ, Oluboyede $Y$, et al. A randomised $2 \times 2$ trial of community versus hospital pulmonary rehabilitation, followed by telephone or conventional follow-up. Health Technol Assess 2010;14:i-xi, 1

6 van Wetering CR, Hoogendoorn M, Mol SJ, et al. Short- and long-term efficacy of a community-based COPD management programme in less advanced COPD: a randomised controlled trial. Thorax 2010;65:7-13.

7 Sewell L, Singh SJ, Williams JE, et al. How long should outpatient pulmonary rehabilitation be? A randomised controlled trial of 4 weeks versus 7 weeks. Thorax 2006;61:767-71.

8 Dodd JW, Hogg L, Nolan J, et al. The COPD assessment test (CAT): response to pulmonary rehabilitation. A multicentre, prospective study. Thorax 2011;66:425-9. 
9 Probst VS, Hernandes NA, Teixeira DC, et al. Reference values for the incremental shuttle walking test. Respir Med 2012;106:243-8.

10 Singh SJ, Jones PW, Evans R, et al. Minimum clinically important improvement for the incremental shuttle walking test. Thorax 2008;63:775-7.

11 Evans RA, Singh SJ, Collier R, et al. Pulmonary rehabilitation is successful for COPD irrespective of MRC dyspnoea grade. Respir Med 2009;103:1070-5.

12 Man WD, Polkey MI, Donaldson N, et al. Community pulmonary rehabilitation after hospitalisation for acute exacerbations of chronic obstructive pulmonary disease: randomised controlled study. BMJ 2004;329:1209.

13 Man WD, Grant A, Hogg L, et al. Pulmonary rehabilitation in patients with MRC Dyspnoea Scale 2. Thorax 2011;66:263.

14 American Thoracic Society. Pulmonary rehabilitation-1999. Am J Respir Crit Care Med 1999;159(5 Pt 1):1666-82.

15 Adams SG, Smith PK, Allan PF, et al. Systematic review of the chronic care model in chronic obstructive pulmonary disease prevention and management. Arch Intern Med 2007:167: 551-61.

16 Wagg K. Unravelling self-management for COPD: what next? Chron Respir Dis 2012;9:5-7.

17 Griffiths TL, Phillips CJ, Davies S, et al. Cost effectiveness of an outpatient multidisciplinary pulmonary rehabilitation programme. Thorax 2001:56:779-84.

18 British Thoracic Society and the Primary Care Respiratory Society UK. IMPRESS Guide to Pulmonary Rehabilitation. 2011, Report No.: Vol 3. 